\title{
Isoniazid Preventive Therapy in HIV-Infected Pregnant and Postpartum Women
}

\author{
A. Gupta, G. Montepiedra, L. Aaron, G. Theron, K. McCarthy, S. Bradford, \\ T. Chipato, T. Vhembo, L. Stranix-Chibanda, C. Onyango-Makumbi, \\ G.R. Masheto, A. Violari, B.T. Mmbaga, L. Aurpibul, R. Bhosale, \\ V. Mave, V. Rouzier, A. Hesseling, K. Shin, B. Zimmer, D. Costello, \\ T.R. Sterling, N. Chakhtoura, P. Jean-Philippe, and A. Weinberg, \\ for the IMPAACT P1078 TB APPRISE Study Team*
}

A BSTRACT

\section{BACKGROUND}

The safety, efficacy, and appropriate timing of isoniazid therapy to prevent tuberculosis in pregnant women with human immunodeficiency virus (HIV) infection who are receiving antiretroviral therapy are unknown.

METHODS

In this multicenter, double-blind, placebo-controlled, noninferiority trial, we randomly assigned pregnant women with HIV infection to receive isoniazid preventive therapy for 28 weeks, initiated either during pregnancy (immediate group) or at week 12 after delivery (deferred group). Mothers and infants were followed through week 48 after delivery. The primary outcome was a composite of treatment-related maternal adverse events of grade 3 or higher or permanent discontinuation of the trial regimen because of toxic effects. The noninferiority margin was an upper boundary of the $95 \%$ confidence interval for the between-group difference in the rate of the primary outcome of less than 5 events per 100 person-years.

\section{RESULTS}

A total of 956 women were enrolled. A primary outcome event occurred in 72 of 477 women (15.1\%) in the immediate group and in 73 of 479 (15.2\%) in the deferred group (incidence rate, 15.03 and 14.93 events per 100 person-years, respectively; rate difference, 0.10 ; $95 \%$ confidence interval [CI], -4.77 to 4.98 , which met the criterion for noninferiority). Two women in the immediate group and 4 women in the deferred group died (incidence rate, 0.40 and 0.78 per 100 person-years, respectively; rate difference, -0.39 ; $95 \% \mathrm{CI},-1.33$ to 0.56 ); all deaths occurred during the postpartum period, and 4 were from liver failure ( 2 of the women who died from liver failure had received isoniazid [1 in each group]). Tuberculosis developed in 6 women ( 3 in each group); the incidence rate was 0.60 per 100 person-years in the immediate group and 0.59 per 100 personyears in the deferred group (rate difference, $0.01 ; 95 \%$ CI, -0.94 to 0.96 ). There was a higher incidence in the immediate group than in the deferred group of an event included in the composite adverse pregnancy outcome (stillbirth or spontaneous abortion, low birth weight in an infant, preterm delivery, or congenital anomalies in an infant) ( $23.6 \%$ vs. $17.0 \%$; difference, 6.7 percentage points; $95 \%$ CI, 0.8 to 11.9 ).

\section{CONCLUSIONS}

The risks associated with initiation of isoniazid preventive therapy during pregnancy appeared to be greater than those associated with initiation of therapy during the postpartum period. (Funded by the National Institutes of Health; IMPAACT P1078 TB APPRISE ClinicalTrials.gov number, NCT01494038.)
The authors' full names, academic degrees, and affiliations are listed in the Appendix. Address reprint requests to Dr. Gupta at the Center for Clinical Global Health Education, Division of Infectious Diseases, Johns Hopkins University School of Medicine, 600 North Wolfe St., Phipps 540B, Baltimore, MD 21287, or at agupta25@jhmi.edu.

*A list of members of the IMPAACT P1078 TB APPRISE Study Team is provided in the Supplementary Appendix, available at NEJM.org.

N EnglJ Med 2019;381:1333-46. DOI: 10.1056/NEJMoal 1813060 Copyright (@) 2019 Massachusetts Medical Society. 
T UBERCULOSIS IS THE LEADING CAUSE OF health complications and death among persons with human immunodeficiency virus (HIV) infection who reside in low-income and middle-income countries with a high tuberculosis burden. ${ }^{1}$ Among women, tuberculosis predominantly affects those of reproductive age. When tuberculosis develops during pregnancy or the early postpartum period, it is associated with adverse maternal, pregnancy, and infant outcomes..$^{2-7}$ There is consensus regarding the net benefit of treating active tuberculosis during pregnancy and providing isoniazid to prevent active tuberculosis in persons with HIV, particularly in persons who have latent tuberculosis infection. However, safety and efficacy data are lacking regarding isoniazid preventive therapy in pregnant women who are receiving antiretroviral therapy (ART), because pregnant women have been consistently excluded from trials of isoniazid preventive therapy. ${ }^{8-14}$

Many physiological changes that occur during pregnancy and the postpartum period affect the immune system and the way drugs are absorbed, distributed, metabolized, and eliminated. ${ }^{15-17}$ Data from small, retrospective studies $^{18,19}$ suggest that women who are pregnant or who have given birth in the previous 3 months have a higher risk of adverse events and druginduced liver injury, including from isoniazid, than women who are not pregnant or who have not recently given birth. ${ }^{16,20}$ World Health Organization (WHO) guidelines recommend initiation of isoniazid preventive therapy in pregnant women with HIV on the basis of data from nonpregnant adults. ${ }^{14}$ The primary objective of the International Maternal Pediatric Adolescent AIDS Clinical Trials (IMPAACT) Network P1078 TB APPRISE trial (TB Ante vs. Postpartum Prevention with INH in HIV Seropositive Mothers and Their Exposed Infants) was to evaluate the safety of initiating isoniazid preventive therapy during pregnancy, as compared with initiating it during the postpartum period, in women with HIV who are living in areas with a high tuberculosis burden. We hypothesized that initiating isoniazid preventive therapy during pregnancy would be as safe as deferring therapy to week 12 after delivery.
METHODS

\section{TRIAL DESIGN AND TREATMENT}

We conducted a prospective, double-blind, placebocontrolled, randomized, noninferiority trial at 13 sites in eight countries that have a high prevalence of tuberculosis ( $\geq 60$ cases per 100,000 population). Participants were randomly assigned to begin taking oral isoniazid either during pregnancy (immediate group) or at week 12 after delivery (deferred group). The immediate group received isoniazid (300 mg daily) from the time of trial entry through 28 weeks after enrollment and then received placebo until week 40 after delivery. The deferred group received placebo from the time of trial entry until week 12 after delivery and then received isoniazid (300 mg daily) for 28 weeks. All women received locally supplied, open-label pyridoxine (vitamin $\mathrm{B}_{6}$ ) and a prenatal multivitamin from the time of trial entry until week 40 after delivery. Randomization was stratified according to the duration of gestation at trial entry ( $\geq 14$ weeks to $<24$ weeks or $\geq 24$ weeks to $\leq 34$ weeks) and was balanced at each site. Full details of the trial design and conduct are provided in the protocol and the statistical analysis plan, available with the full text of this article at NEJM.org.

\section{PARTICIPANTS}

Eligible participants were pregnant women, at 14 weeks through 34 weeks of gestation, who had HIV infection and were 18 years of age or older, weighed at least $35 \mathrm{~kg}$, and had an absolute neutrophil count of 750 cells or more per cubic millimeter, a hemoglobin level of $7.5 \mathrm{~g}$ or more per deciliter, a platelet count of 50,000 or more per cubic millimeter, and levels of aspartate aminotransferase (AST), alanine aminotransferase (ALT), and total bilirubin that were no more than 1.25 times the upper limit of the normal range within 30 days before trial entry. Exclusion criteria were suspected active tuberculosis, recent known tuberculosis exposure, treatment for tuberculosis for more than 30 days in the previous year, evidence of recent acute hepatitis, or peripheral neuropathy of grade 1 or higher. Evidence of latent tuberculosis infection was not required for entry. 


\section{TRIAL OVERSIGHT}

All women provided written informed consent. The trial was approved by local and collaborating institutional review boards and was reviewed every 6 months by an independent data and safety monitoring board (see the Supplementary Appendix, available at NEJM.org). The isoniazid and placebo were supplied by the Clinical Research Products Management Center of the National Institute of Allergy and Infectious Diseases. The isoniazid, placebo, and testing kits were purchased with trial funds; there was no commercial support for the trial. In February 2016, in response to a request by the data and safety monitoring board, a letter about potential risks of receiving isoniazid preventive therapy in addition to ART was issued to all participants after two participants in the trial died from fulminant liver failure.

The data were gathered at the local sites; the IMPAACT Statistical and Data Analysis Center analyzed the data according to the statistical analysis plans. All the authors vouch for the accuracy and completeness of the data and analyses and for the fidelity of the trial to the protocol.

\section{TRIAL PROCEDURES}

Baseline serologic testing for HIV antibodies, an assessment of CD4 count, an HIV type 1 quantitative RNA assay, testing for hepatitis $\mathrm{C}$ virus antibody and hepatitis B surface antigen, an assessment of creatinine and glucose levels, and an interferon gamma release assay (IGRA) for tuberculosis (with the use of the QuantiFERON-TB Gold test ${ }^{21}$ ) were performed at entry. Follow-up visits occurred every 4 weeks during pregnancy and at labor and delivery; follow-up of mothers and infants occurred every 4 weeks after delivery for 48 weeks. Assessments included a medical history, physical examination, complete blood count, measurement of the ALT level (AST and total bilirubin levels were also assessed if there was an elevation in ALT level of grade 1 or higher), assessment of tuberculosis exposure or disease (with acid-fast bacilli smear and Mycobacterium tuberculosis culture performed if tuberculosis was suspected), maternal peripheral neuropathy screening, ${ }^{22}$ and HIV testing of the infant. Adherence to the trial regimen was assessed monthly on the basis of patient-reported adherence and pill counts.

\section{PRIMARY OUTCOME}

The primary outcome was a composite safety outcome of maternal adverse events of grade 3 or higher that were possibly, probably, or definitely related to isoniazid or placebo or permanent discontinuation of the trial regimen because of toxic effects, whichever occurred first, through week 48 after delivery. Members of an independent end-point review committee, who were unaware of the trial-group assignments, determined whether adverse events were related to isoniazid or placebo. Hepatotoxicity and peripheral neuropathy were graded with the use of protocoldefined criteria. All other adverse events were graded with the use of the Table for Grading the Severity of Adult and Pediatric Adverse Events of the Division of AIDS. ${ }^{23}$

\section{SECONDARY OUTCOMES}

Key secondary maternal outcomes were adverse events of any cause of grade 3 or higher, hepatotoxicity, death, and tuberculosis, assessed through week 48 after delivery. Hepatotoxicity was defined as an elevation of grade 3 or higher in liver-enzyme levels (ALT, AST, or total bilirubin); an elevation of grade 2 or higher in total bilirubin and ALT levels; or an elevation of grade 2 or higher in ALT level with symptomatic clinical hepatitis. Key infant outcomes were adverse events of grade 3 or higher, tuberculosis, and death, assessed through week 48 after birth. The composite efficacy outcome was maternal tuberculosis, infant tuberculosis, maternal death, infant death, or stillbirth or spontaneous abortion. Diagnosis of tuberculosis was categorized as probable or confirmed according to standardized criteria, and cases were reviewed by an independent end-point review committee composed of five experts in maternal and pediatric tuberculosis who were unaware of the trial-group assignments.

The composite adverse pregnancy outcome, a key secondary outcome, was stillbirth (fetal death at 20 weeks of gestation or later) or spontaneous abortion (loss of pregnancy before 20 weeks of gestation), low birth weight $(<2500 \mathrm{~g})$ in an in- 
fant, preterm delivery (delivery before 37 weeks of gestation, with duration of gestation determined with the use of the Ballard examination when available or by obstetrical estimate), or major congenital anomalies in an infant (defined according to the Metropolitan Atlanta Congenital Defects Program of the Centers for Disease Control and Prevention ${ }^{24}$ ); adverse pregnancy outcomes were also assessed individually. The composite severe adverse pregnancy outcome was stillbirth or spontaneous abortion, very low birth weight ( $<1500 \mathrm{~g})$ in an infant, very preterm delivery (delivery before 34 weeks of gestation), or major congenital anomalies in an infant.

\section{STATISTICAL ANALYSIS}

On the basis of previous studies of isoniazid preventive therapy in persons with HIV infection, ${ }^{10-12}$ we assumed that the incidence rate of the primary outcome would be 5 events per 100 person-years in the deferred group. Noninferiority of immediate isoniazid preventive therapy to deferred therapy with respect to the primary outcome would be shown if the upper boundary of the $95 \%$ confidence interval for the betweengroup difference in the incidence rate was less than 5 events per 100 person-years. We calculated that 950 women (475 per group) would need to be enrolled to provide the trial with a power of at least $90 \%$ to show noninferiority of immediate therapy to deferred therapy, at a twosided alpha level of 0.05 (see the Supplementary Appendix).

All analyses were prespecified unless otherwise noted. The primary analysis was performed on an intention-to-treat basis and included all women who underwent randomization, with data censored at the last trial visit. We performed a post hoc worst-case sensitivity analysis, in which participants who did not complete follow-up were considered as having had a primary outcome event, and a post hoc complete-case sensitivity analysis, which included only participants who had data available through week 48 after delivery. We also performed a per-protocol analysis, which included all eligible enrolled women who completed the trial regimen according to the protocol, all women in whom tuberculosis developed or who died during the treatment period, and all women who permanently discontinued the trial regimen because they met criteria for discontinuation as specified in the protocol. The absolute difference between groups in the rate of the primary outcome was calculated with the use of Mantel-Haenszel estimates, stratified according to duration of gestation at trial entry; confidence intervals were calculated according to the method of Greenland and Robins, ${ }^{25}$ and the significance level was set at $5 \%$, given that sequential monitoring with a Haybittle-Peto use function boundary for the alpha level was used for interim analyses. ${ }^{26}$ The percentage of participants with outcome events and the betweengroup differences in the percentage, with 95\% confidence intervals, were also computed. The efficacy outcomes of maternal tuberculosis and infant tuberculosis and the composite efficacy outcome were assessed in all mothers who underwent randomization and their infants, except for mothers who underwent randomization but were later found to have had tuberculosis at the time of enrollment. The analysis of the composite efficacy outcome was stratified according to duration of gestation at trial entry.

Within-group incidence rates and $95 \%$ confidence intervals for rate differences between groups were computed for secondary outcomes, except for the composite and individual pregnancy outcomes, which were compared with the use of Fisher's exact test with mid-P adjustment $^{27}$; a two-sided $\mathrm{P}$ value of 0.05 or less was considered to indicate statistical significance. There was no adjustment for multiple testing in the analyses of secondary outcomes.

\section{RESULTS}

\section{TRIAL POPULATION}

From August 2014 through April 2016, a total of 956 participants were enrolled; 477 women were assigned to the immediate group, and 479 to the deferred group (Fig. 1). The baseline characteristics were well balanced between the two groups (Table 1). The median age was 29 years, $90.5 \%$ were black African, $33.6 \%$ had a duration of gestation of 14 weeks to less than 24 weeks, and $30.0 \%$ had positive IGRA results for latent tuberculosis at enrollment. The median CD4 count was 493 cells per cubic millimeter, and all participants except one were receiving highly active ART; in $85.1 \%$ of all participants, the regimen included efavirenz. A total of 171 women (17.9\%) discontinued the trial prematurely: 6 women $(0.6 \%)$ died ( 2 women in the immediate group 
and 4 in the deferred group); 78 women (8.2\%) withdrew consent (44 women in the immediate group and 34 in the deferred group); 1 woman $(0.1 \%)$, in the deferred group, was not eligible; 1 woman $(0.1 \%)$, in the immediate group, enrolled in another study; and 85 women (8.9\%) were lost to follow-up (41 women in the immediate group and 44 in the deferred group). The time to the last clinic visit was balanced between the two groups (Fig. S1 in the Supplementary Appendix). Data on adherence to isoniazid treatment were available for 466 participants in the immediate group and 413 participants in the deferred group. According to patient-reported adherence data, 400 women $(85.8 \%)$ in the immediate group and $363(87.9 \%)$ in the deferred group completed at least $90 \%$ of the treatment ( $\mathrm{P}=0.43$ by Fisher's exact test); according to assessment of pill count, 408 of 463 women $(88.1 \%)$ in the immediate group and 376 of $413(91.0 \%)$ in the deferred group completed at least $90 \%$ of the treatment ( $\mathrm{P}=0.19$ by Fisher's exact test).

\section{PRIMARY OUTCOME}

In the intention-to-treat population, a primary outcome event occurred in 72 women $(15.1 \%)$ in the immediate group and in $73(15.2 \%)$ in the deferred group (incidence rate, 15.03 and 14.93 events per 100 person-years, respectively; rate difference, $0.10 ; 95 \% \mathrm{CI},-4.77$ to 4.98 ) (Table 2). These results met the criterion for noninferiority of immediate treatment to deferred treatment. The results of the per-protocol analysis were consistent with these findings and also showed noninferiority. Treatment-related maternal adverse events of grade 3 or higher and the numbers and percentages of participants who discontinued the trial regimen because of toxic effects are shown in Table 3. Additional data on incidence rates in the intention-to-treat and perprotocol populations according to duration of gestation, reasons for discontinuation of the trial regimen, and estimated percentages of participants with primary and secondary outcome events are provided in Tables S1 through S3 in the Supplementary Appendix.

The results of sensitivity analyses for missing data did not show noninferiority of immediate treatment to deferred treatment. In the worstcase analysis, the between-group difference in the incidence rate was 1.45 events per 100 personyears ( $95 \%$ confidence interval [CI], -5.49 to 8.4 ),

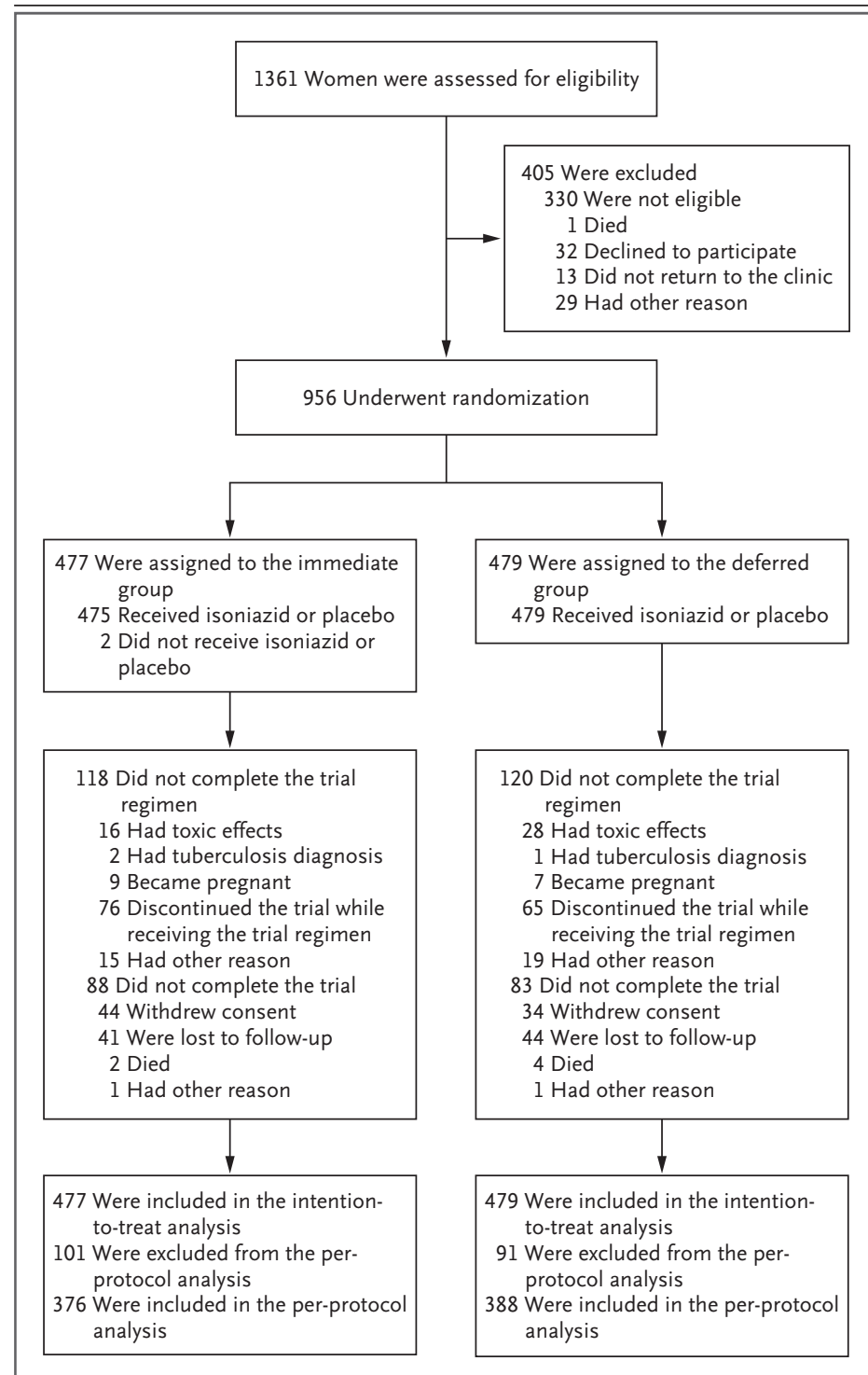

Figure 1. Enrollment, Randomization, and Analysis.

Participants were assigned to receive 28 weeks of isoniazid preventive therapy initiated either during pregnancy (immediate group) or at 12 weeks after delivery (deferred group). Participants were excluded from the per-protocol analysis for one or more of the following reasons: they withdrew consent, they were lost to follow-up, they did not complete the trial regimen according to the protocol, or they had been found after randomization to be ineligible.

and in the complete-case analysis, the betweengroup difference in the incidence rate was 0.21 events per 100 person-years ( $95 \% \mathrm{CI},-5.34$ to 5.75) (Table S4 in the Supplementary Appendix).

\section{SECONDARY MATERNAL SAFETY OUTCOMES}

Intention-to-treat analyses showed no significant differences in the incidence of adverse events of 


\begin{tabular}{|c|c|c|}
\hline Characteristic & $\begin{array}{l}\text { Immediate Group } \\
\qquad(N=477)\end{array}$ & $\begin{array}{l}\text { Deferred Group } \\
\qquad(\mathrm{N}=479)\end{array}$ \\
\hline Median age (IQR) $-y r$ & $29(25-33)$ & $29(24-33)$ \\
\hline \multicolumn{3}{|l|}{ Ethnic group — no. $(\%) \dagger$} \\
\hline Black African & $436(91.4)$ & $429(89.6)$ \\
\hline Black, of African origin & $8(1.7)$ & $15(3.1)$ \\
\hline Indian or South Asian & $17(3.6)$ & $15(3.1)$ \\
\hline Thai & $10(2.1)$ & $12(2.5)$ \\
\hline Other Asian & $5(1.0)$ & $8(1.7)$ \\
\hline Mixed, of predominantly African ancestry & $1(0.2)$ & 0 \\
\hline \multicolumn{3}{|l|}{ Country - no. (\%) } \\
\hline Botswana & $60(12.6)$ & $60(12.5)$ \\
\hline Haiti & $8(1.7)$ & $15(3.1)$ \\
\hline India & $17(3.6)$ & $16(3.3)$ \\
\hline South Africa & $91(19.1)$ & $91(19.0)$ \\
\hline Tanzania & $41(8.6)$ & $39(8.1)$ \\
\hline Thailand & $15(3.1)$ & $18(3.8)$ \\
\hline Uganda & $83(17.4)$ & $83(17.3)$ \\
\hline Zimbabwe & $162(34.0)$ & $157(32.8)$ \\
\hline \multicolumn{3}{|l|}{ Gestation - no. (\%) } \\
\hline 14 to $<24 \mathrm{wk}$ & $161(33.8)$ & $160(33.4)$ \\
\hline 24 to $\leq 34 \mathrm{wk}$ & $316(66.2)$ & $319(66.6)$ \\
\hline Median body-mass index (IQR) $\ddagger$ & $26(23-30)$ & $26(23-30)$ \\
\hline Median CD4 count (IQR) - cells $/ \mathrm{mm}^{3}$ & $491(351-670)$ & $496(355-669)$ \\
\hline $\begin{array}{l}\text { HIV RNA level below the lower limit of quantification } \\
\qquad \text { - no./total no. (\%) }\end{array}$ & $303 / 476(63.7)$ & $299 / 478(62.6)$ \\
\hline WHO clinical stage $1-$ no. (\%) $\int$ & $426(89.3)$ & $419(87.5)$ \\
\hline $\begin{array}{l}\text { Receipt of highly active antiretroviral therapy at entry } \\
\qquad \text { - no. (\%) }\end{array}$ & $476(99.8)$ & $479(100.0)$ \\
\hline Efavirenz-tenofovir-emtricitabine or lamivudine & $401(84.1)$ & $401(83.7)$ \\
\hline Efavirenz-zidovudine-lamivudine & $3(0.6)$ & $8(1.7)$ \\
\hline $\begin{array}{l}\text { Nevirapine-zidovudine or tenofovir-emtricitabine } \\
\text { or lamivudine }\end{array}$ & $59(12.4)$ & $61(12.7)$ \\
\hline $\begin{array}{l}\text { Lopinavir or atazanavir-ritonavir with tenofovir } \\
\text { or zidovudine-emtricitabine or lamivudine }\end{array}$ & $13(2.7)$ & $9(1.9)$ \\
\hline Receipt of efavirenz only at entry — no. (\%) & $1(0.2)$ & 0 \\
\hline Positive IGRA status — no./total no. (\%) & $139 / 470(29.6)$ & $144 / 472(30.5)$ \\
\hline $\begin{array}{l}\text { Positive test for hepatitis B surface antigen } \\
\quad \text { - no./total no. (\%) }\end{array}$ & $15 / 476(3.2)$ & $18 / 479(3.8)$ \\
\hline Positive serologic test for hepatitis C — no./total no. (\%) & $5 / 449(1.1)$ & $4 / 445(0.9)$ \\
\hline
\end{tabular}

* Percentages may not total 100 because of rounding. HIV denotes human immunodeficiency virus, IGRA interferon gamma release assay, and IQR interquartile range.

$\uparrow$ Ethnic group was reported by the participants and categorized by trained staff according to categories defined by the Division of AIDS of the National Institute of Allergy and Infectious Diseases.

The body-mass index is the weight in kilograms divided by the square of the height in meters.

$\int$ World Health Organization (WHO) stage 1 indicates asymptomatic HIV infection. 
Table 2. Primary Safety Outcome and Secondary Maternal and Infant Outcomes through Week 48 after Delivery.*

\begin{tabular}{|c|c|c|c|c|c|}
\hline \multirow[t]{2}{*}{ Outcome } & \multicolumn{2}{|c|}{ Immediate Group } & \multicolumn{2}{|c|}{ Deferred Group } & \multirow[t]{2}{*}{$\begin{array}{c}\text { Incidence Rate } \\
\text { Difference }(95 \% \mathrm{Cl})\end{array}$} \\
\hline & no./total no. (\%) & $\begin{array}{c}\text { incidence rate per } \\
100 \text { person- } y r\end{array}$ & no./total no. (\%) & $\begin{array}{c}\text { incidence rate per } \\
100 \text { person- } y r\end{array}$ & \\
\hline \multicolumn{6}{|l|}{ Primary outcome $\dagger$} \\
\hline Intention-to-treat population & $72 / 477(15.1)$ & 15.03 & $73 / 479(15.2)$ & 14.93 & $0.10(-4.77$ to 4.98$)$ \\
\hline Per-protocol population & $64 / 376(17.0)$ & 16.00 & $69 / 388(17.8)$ & 16.71 & $-0.71(-6.27$ to 4.85$)$ \\
\hline \multicolumn{6}{|l|}{ Secondary maternal outcomes } \\
\hline Any grade 3 or 4 adverse event & $144 / 477(30.2)$ & 34.95 & $136 / 479(28.4)$ & 31.26 & $3.69(-4.07$ to 11.45$)$ \\
\hline Hepatotoxicity & $29 / 477(6.1)$ & 5.80 & $34 / 479(7.1)$ & 6.69 & -0.89 ( -3.98 to 2.19$)$ \\
\hline Peripheral neuropathy & $1 / 477(0.2)$ & 0.19 & $0 / 479(0.0)$ & 0.00 & $0.19(-0.19$ to 0.57$)$ \\
\hline Death & $2 / 477(0.4)$ & 0.40 & $4 / 479(0.8)$ & 0.78 & $-0.39(-1.33$ to 0.56$)$ \\
\hline $\begin{array}{l}\text { Death due to hepatitis after } \\
\text { receiving isoniazid』 }\end{array}$ & $1 / 477(0.2)$ & 0.20 & $1 / 479(0.2)$ & 0.20 & $0.00(-0.54$ to 0.55$)$ \\
\hline $\begin{array}{l}\text { Permanent discontinuation of trial } \\
\text { regimen because of toxic } \\
\text { effects } \mathbb{}\end{array}$ & $16 / 477(3.4)$ & 3.16 & $28 / 479(5.8)$ & 5.48 & $-2.32(-4.88$ to 0.23$)$ \\
\hline Tuberculosis 9 & $3 / 477(0.6)$ & 0.60 & $3 / 478(0.6)$ & 0.59 & $0.01(-0.94$ to 0.96$)$ \\
\hline \multicolumn{6}{|l|}{ Secondary infant outcomes } \\
\hline Any grade 3 or 4 adverse event & $191 / 445$ (42.9) & 70.74 & $192 / 464(41.4)$ & 65.75 & 4.99 (-8.69 to 18.67$)$ \\
\hline HIV infection & $3 / 439(0.7)$ & 0.79 & $7 / 458(1.5)$ & 1.75 & $-0.96(-2.54$ to 0.61$)$ \\
\hline Infant death: 0-48 wk after birth & $11 / 445(2.5)$ & 2.99 & $17 / 464(3.7)$ & 4.42 & $-1.43(-4.17$ to 1.32$)$ \\
\hline Neonatal death: $0-7$ days after birth\| & $4 / 445(0.9)$ & - & $5 / 464(1.1)$ & - & - \\
\hline Tuberculosis*** & $0 / 445(0.0)$ & 0.54 & $1 / 464(0.2)$ & 0.52 & $0.02(-1.02$ to 1.07$)$ \\
\hline
\end{tabular}

* All analyses were performed on an intention-to-treat basis unless otherwise specified. Incidence rates were used to summarize the outcomes, given that the follow-up period of observation was expected to vary from 48 weeks to 74 weeks, depending on the duration of gestation at entry. The noninferiority margin was an upper boundary of the $95 \%$ confidence interval for the difference in the rate of the primary outcome between the two groups of less than 5 events per 100 person-years.

$\dagger \quad$ The primary safety outcome was a maternal adverse event of grade 3 or higher that was possibly, probably, or definitely related to isoniazid or placebo (as adjudicated by an independent end-point review committee, whose members were unaware of the trial-group assignments) or permanent discontinuation of the trial regimen because of toxic effects, whichever occurred earlier. The intention-to-treat population included all women who underwent randomization. The per-protocol population included all eligible enrolled women who completed the trial regimen according to the protocol, who died during the treatment period, and who permanently discontinued the trial regimen because they met criteria for discontinuation as specified in the protocol, and all women in whom tuberculosis developed.

$t$ Hepatotoxicity was defined as an elevation of grade 3 or higher in liver-enzyme levels (alanine aminotransferase [ALT], aspartate aminotransferase, or total bilirubin); a grade 2 or higher elevation in total bilirubin and ALT levels; or a grade 2 or higher elevation in ALT level with symptomatic clinical hepatitis.

$\int$ The analysis of this outcome was a post hoc analysis. Additional details are provided in Table S5 in the Supplementary Appendix.

I Of the six cases of tuberculosis adjudicated by the independent end-point review committee, four were confirmed cases of pulmonary tuberculosis and two were probable cases of pulmonary tuberculosis. Three cases were in women who had positive IGRA status at entry, one case was in a woman with indeterminate status at entry, and one case was in a woman whose IGRA status changed from negative to positive before tuberculosis diagnosis (Table S6 in the Supplementary Appendix).

$\|$ Incidence rates are not reported for this category because of the short follow-up time.

w* The calculation of the incidence rates in this category includes deaths from unknown causes (two in the immediate group and one in the deferred group).

any cause (treatment-related or not) of grade 3 or higher (Tables 2 through 4). The incidence rate of any grade 3 or 4 adverse event related to isoniazid or placebo was 34.95 per 100 person-years in the immediate group and 31.26 per 100 personyears in the deferred group (rate difference, 3.69;
$95 \% \mathrm{CI},-4.07$ to 11.45$)$. The incidence rate of hepatotoxicity was 5.80 and 6.69 per 100 personyears, respectively (rate difference, $-0.89 ; 95 \%$ CI, -3.98 to 2.19 ).

Six women died -2 women in the immediate group and 4 in the deferred group (incidence 


\begin{tabular}{|c|c|c|c|}
\hline \multirow[t]{2}{*}{ Event } & $\begin{array}{l}\text { Immediate Group } \\
\quad(\mathrm{N}=477)\end{array}$ & $\begin{array}{l}\text { Deferred Group } \\
\qquad(\mathrm{N}=479)\end{array}$ & $\begin{array}{c}\text { Total } \\
(\mathrm{N}=956)\end{array}$ \\
\hline & \multicolumn{3}{|c|}{ number of participants (percent) } \\
\hline Any treatment-related grade $\geq 3$ adverse event & $70(15)$ & $70(15)$ & $140(15)$ \\
\hline Grade $\geq 3$ abnormal laboratory findings & $27(6)$ & $36(8)$ & $63(7)$ \\
\hline Abnormal laboratory chemical values & $26(5)$ & $34(7)$ & $60(6)$ \\
\hline Elevated liver-enzyme levels & $26(5)$ & $33(7)$ & $59(6)$ \\
\hline Abnormal hematologic values & $1(<1)$ & $3(1)$ & $4(<1)$ \\
\hline Grade $\geq 3$ clinical adverse event & $50(10)$ & $41(9)$ & $91(10)$ \\
\hline Gastrointestinal event & $2(<1)$ & $1(<1)$ & $3(<1)$ \\
\hline Hepatitis & $4(1)$ & $5(1)$ & $9(1)$ \\
\hline Nervous system disorder & $1(<1)$ & $2(<1)$ & $3(<1)$ \\
\hline Peripheral neuropathy & $1(<1)$ & 0 & $1(<1)$ \\
\hline $\begin{array}{l}\text { Event related to pregnancy, the puerperium, or } \\
\text { the perinatal period }\end{array}$ & $15(3)$ & $13(3)$ & $28(3)$ \\
\hline Eclampsia or preeclampsia & $5(1)$ & $3(1)$ & $8(1)$ \\
\hline Gestational hypertension & $1(<1)$ & $2(<1)$ & $3(<1)$ \\
\hline Hemorrhage & $1(<1)$ & $1(<1)$ & $2(<1)$ \\
\hline Stillbirth or spontaneous abortion $†$ & $6(1)$ & $2(<1)$ & $8(1)$ \\
\hline Weight losst & $30(6)$ & $24(5)$ & $54(6)$ \\
\hline Other clinical adverse event & $5(1)$ & $2(<1)$ & $7(1)$ \\
\hline $\begin{array}{l}\text { Permanent discontinuation of trial regimen because } \\
\text { of toxic effects, but no treatment-related } \\
\text { grade } \geq 3 \text { adverse events }\end{array}$ & $2(<1)$ & $3(<1)$ & $5(<1)$ \\
\hline $\begin{array}{l}\text { Treatment-related adverse events were events that w } \\
\text { Stillbirth was defined as fetal death at } 20 \text { weeks of ge } \\
\text { nancy before } 20 \text { weeks of gestation. } \\
\text { Weight loss was defined as unintentional loss of at le }\end{array}$ & $\begin{array}{l}\text { e possibly, probably } \\
\text { tation or later. Spon } \\
\text { st } 10 \% \text { of body wei }\end{array}$ & $\begin{array}{l}\text { definitely relate } \\
\text { ous abortion w } \\
\text { see the Suppler }\end{array}$ & $\begin{array}{l}\text { azid or pla } \\
\text { as loss o } \\
\text { ppendix). }\end{array}$ \\
\hline
\end{tabular}

rate, 0.40 and 0.78 per 100 person-years, respectively; rate difference, -0.39 ; $95 \% \mathrm{CI},-1.33$ to 0.56) (Table 2, and Table S5 in the Supplementary Appendix). The primary cause of death in 4 of the women ( 1 in the immediate group and 3 in the deferred group) was liver failure, and all the deaths occurred during the postpartum period; 2 women had received isoniazid (1 woman in each group), and all 4 were receiving efavirenztenofovir-emtricitabine at the time of death.

There were no noted imbalances between the groups in the prevalence of peripheral neuropathy or hepatotoxicity (Table 2 and Fig. 2A). Symptomatic hepatitis developed in 11 women $(1.2 \%)-3$ women in the immediate group and 8 in the deferred group. All cases of symptomatic hepatitis occurred during the postpartum period; at the time of the event, 4 women were receiving isoniazid, and 4 women had discontinued isoniazid at 2.4 weeks, 4.9 weeks, 14.7 weeks, and 15.7 weeks before symptom onset. A total of 63 women had elevations in liver-enzyme levels of grade 3 or higher or symptomatic hepatitis (or both); at the time of the first event, 32 women were receiving isoniazid in addition to efavirenzbased ART, 22 were receiving efavirenz-based ART only, and 6 were receiving isoniazid in addition to non-efavirenz-based ART.

\section{ADVERSE PREGNANCY OUTCOMES AND OUTCOMES IN INFANTS}

There were 926 deliveries. A greater percentage of women in the immediate group than in the deferred group had a composite adverse preg- 


\begin{tabular}{|c|c|c|c|}
\hline Event & $\begin{array}{l}\text { Immediate Group } \\
\quad(N=477)\end{array}$ & $\begin{array}{l}\text { Deferred Group } \\
\qquad(\mathrm{N}=479)\end{array}$ & $\begin{array}{c}\text { Total } \\
(\mathrm{N}=956)\end{array}$ \\
\hline & \multicolumn{3}{|c|}{ number of participants (percent) } \\
\hline Any grade $\geq 3$ adverse event & $144(30)$ & $136(28)$ & $280(29)$ \\
\hline Any grade $\geq 3$ serious adverse event $*$ & $69(14)$ & $66(14)$ & $135(14)$ \\
\hline Grade $\geq 3$ abnormal laboratory findings & $63(13)$ & $59(12)$ & $122(13)$ \\
\hline Abnormal laboratory chemical values & $34(7)$ & $40(8)$ & $74(8)$ \\
\hline Elevated liver-enzyme levels & $29(6)$ & $34(7)$ & $63(7)$ \\
\hline Abnormal hematologic values & $35(7)$ & $24(5)$ & $59(6)$ \\
\hline Grade $\geq 3$ clinical adverse event & $106(22)$ & $98(20)$ & $204(21)$ \\
\hline Gastrointestinal event & $8(2)$ & $7(1)$ & $15(2)$ \\
\hline Hepatitis & $5(1)$ & $6(1)$ & $11(1)$ \\
\hline Infection & $17(4)$ & $14(3)$ & $31(3)$ \\
\hline Nervous system disorder & $4(1)$ & $5(1)$ & $9(1)$ \\
\hline Peripheral neuropathy & $1(<1)$ & 0 & $1(<1)$ \\
\hline $\begin{array}{l}\text { Event related to pregnancy, the puerperium, } \\
\text { or the perinatal period }\end{array}$ & $41(9)$ & $39(8)$ & $80(8)$ \\
\hline Eclampsia or preeclampsia & $8(2)$ & $4(1)$ & $12(1)$ \\
\hline Gestational hypertension & $4(1)$ & $5(1)$ & $9(1)$ \\
\hline Hemorrhage & $5(1)$ & $7(1)$ & $12(1)$ \\
\hline Stillbirth or spontaneous abortion $\uparrow$ & $18(4)$ & $9(2)$ & $27(3)$ \\
\hline Weight loss & $31(6)$ & $27(6)$ & $58(6)$ \\
\hline Other clinical adverse event & $36(8)$ & $31(6)$ & $67(7)$ \\
\hline
\end{tabular}

* A serious adverse event was defined as any adverse event that resulted in death, a life-threatening condition, inpatient hospitalization, prolongation of an existing hospitalization, or a persistent or clinically significant disability or incapacity. $\dagger$ One event in the immediate group was from a subsequent pregnancy.

nancy outcome event ( $23.6 \%$ vs. $17.0 \%$; difference, 6.7 percentage points; $95 \%$ CI, 0.8 to 11.9 ; $\mathrm{P}=0.01$ ) (Fig. 2B). There was a higher frequency of each of the individual adverse pregnancy outcomes in the immediate group than in the deferred group, but none of the between-group differences were significant. With respect to the composite severe adverse pregnancy outcome, no significant difference was observed between the immediate group and the deferred group (6.3\% vs. $4.6 \%$; difference, 1.7 percentage points; $95 \% \mathrm{CI},-1.3$ to $4.8 ; \mathrm{P}=0.27)$. The results of a post hoc analysis of adverse pregnancy outcomes according to duration of gestation are provided in Figure S3 in the Supplementary Appendix; the analysis was not adjusted for multiple comparisons. Among the 909 infants born alive, no imbalances between groups were noted in adverse events of grade 3 or higher, HIV infection, or death assessed through week 48 after birth (Table 2).

\section{TUBERCULOSIS AND COMPOSITE EFFICACY ANALYSES}

One woman with confirmed tuberculosis at entry was excluded from the analysis of efficacy outcomes. Tuberculosis developed in 6 women ( 3 in each group), and all cases occurred during the postpartum period (Table 2, and Table S6 in the Supplementary Appendix). The incidence rate of maternal tuberculosis was 0.60 and 0.59 per 100 person-years, respectively (rate difference, 0.01; $95 \%$ CI, -0.94 to 0.96 ). Four cases were confirmed (one was a case of monoresistant [i.e., isoniazid-resistant] tuberculosis), and two cases were probable. There were no cases of tuberculosis in infants in the immediate group, and a probable case of tuberculosis developed in one 
Immediate Treatment Deferred Treatment

A Hepatotoxicity According to Protocol-Specified Definitions

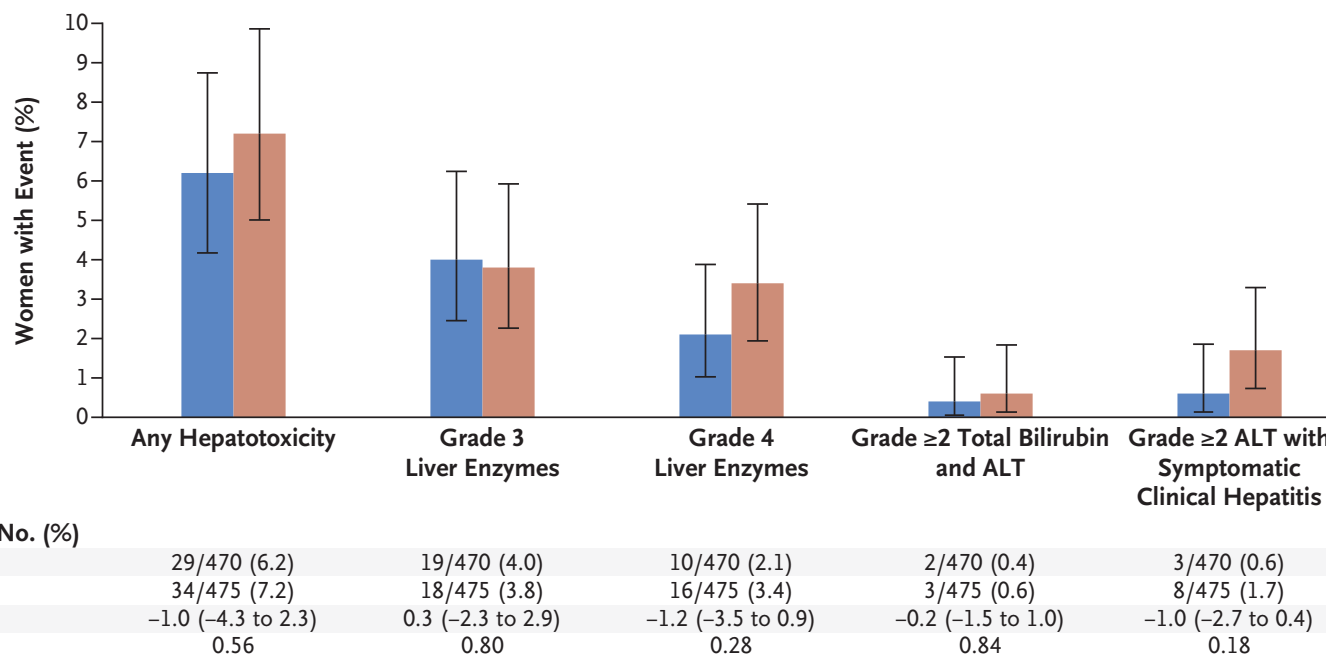

No. of Events/Total No. (\%)

Immediate treatment

Deferred treatment

RD $(95 \% \mathrm{CI})$

0.28

0.18

B Composite Adverse Pregnancy Outcomes and Components

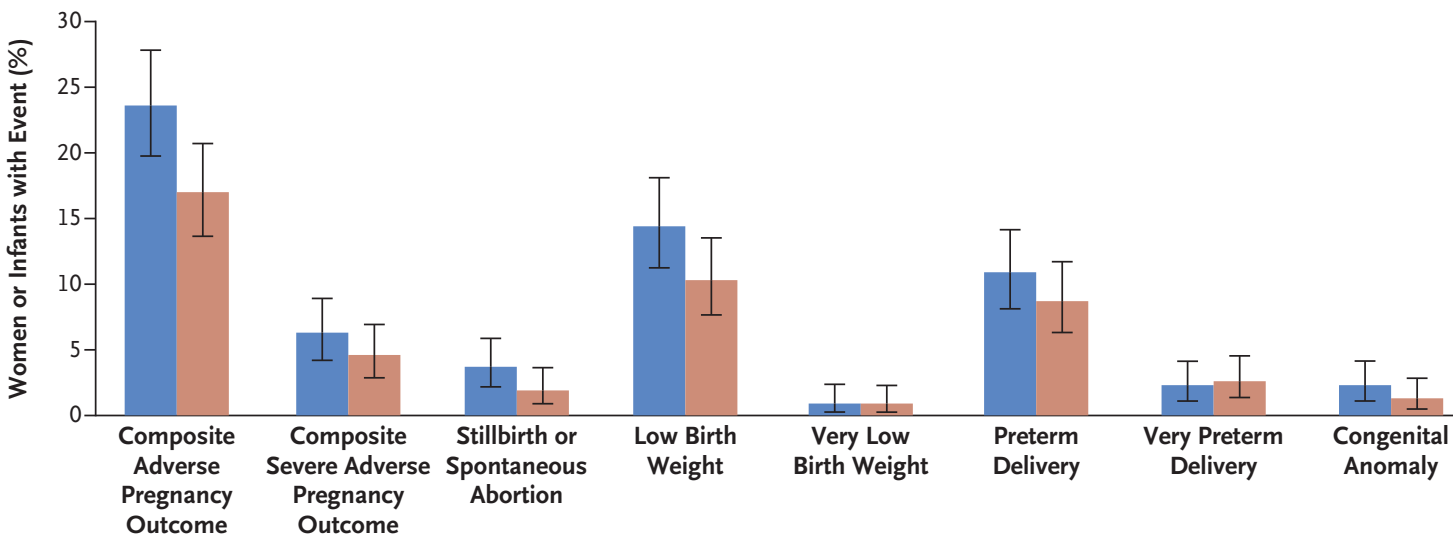

\section{No. of Events/}

Total No. (\%)

Immediate treatment $106 / 449(23.6)$

Deferred treatment

RD $(95 \% \mathrm{CI})$

$78 / 460(17.0)$
$6.7(0.8$ to 11.9$)$

$17 / 459$ (3.7)

$62 / 430(14.4)$

$4 / 430(0.9)$

$48 / 442(10.9)$

$10 / 442(2.3)$

$10 / 440(2.3)$

P Value

0.01

$1.7(-1.3$ to 4.8$)$

$9 / 466(1.9) \quad 46 / 446(10.3)$

$(0.9)$

$2.1(-1.8$ to 6 .

$12 / 458(2.6)$

$6 / 458(1.3)$

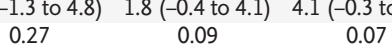

0.86

0.29

0.75

0.26

Figure 2. Hepatotoxicity and Adverse Pregnancy Outcomes.

Hepatotoxicity (Panel A) was defined as a grade 3 or higher elevation in liver-enzyme levels (alanine aminotransferase [ALT], aspartate aminotransferase, or total bilirubin), a grade 2 or higher elevation in total bilirubin and ALT levels, or a grade 2 or higher elevation in ALT level with symptomatic clinical hepatitis. Grading of hepatotoxicity was determined on the basis of criteria specified in the protocol. The composite adverse pregnancy outcome (Panel B) was stillbirth (fetal death at 20 weeks of gestation or later) or spontaneous abortion (loss of pregnancy before 20 weeks of gestation), low birth weight $(<2500 \mathrm{~g})$ in an infant, preterm delivery (delivery before 37 weeks of gestation, with duration of gestation determined with the use of the Ballard examination when available or by obstetrical estimate), or major congenital anomalies in an infant (defined according to the criteria of the Metropolitan Atlanta Congenital Defects Program of the Centers for Disease Control and Prevention ${ }^{24}$ ). The composite severe adverse pregnancy outcome was stillbirth or spontaneous abortion, very low birth weight $(<1500 \mathrm{~g})$ in an infant, very preterm delivery (delivery before 34 weeks of gestation), or major congenital anomalies in an infant. The risk difference (RD) is the between-group difference in percentage points. 
infant in the deferred group at 12 months of age. In an analysis stratified according to duration of gestation at trial entry, there were no between-group differences in the time to the composite efficacy outcome (Fig. S2B in the Supplementary Appendix). Attempts were made to contact the 85 women who were lost to follow-up; we were able to ascertain vital status for 74 of these women $(87.1 \%)$. No maternal deaths, hospitalizations, or tuberculosis cases were reported.

\section{DISCUSSION}

Isoniazid in addition to ART is strongly recommended for the prevention of tuberculosis in patients with HIV infection. ${ }^{14}$ On the basis of data from nonpregnant adults and the absence of harm observed in small studies of isoniazid preventive therapy in pregnancy, ${ }^{28-30}$ the WHO recommends isoniazid preventive therapy and ART during pregnancy, ${ }^{14}$ but it has acknowledged the need for pregnancy-specific data. ${ }^{14}$ This trial showed that isoniazid preventive therapy initiated during pregnancy was noninferior to initiation at 3 months after delivery with respect to maternal adverse events, and there was a low incidence of tuberculosis in both groups. However, with careful clinical monitoring and monitoring of liver function, we observed a higher incidence of maternal treatment-related serious adverse events during the postpartum period than expected in both the immediate group and the deferred group. We also observed a higher incidence of adverse pregnancy outcomes in the immediate group than in the deferred group when analyzed a priori as a composite outcome. No other differences in secondary maternal or infant outcomes were observed between groups.

Risk factors for hepatotoxicity (including druginduced hepatotoxicity) include female sex, pregnancy, delivery within the previous 3 months, and obesity. ${ }^{20,31}$ Retrospective data from the 1980s have suggested a higher risk of toxic effects related to isoniazid treatment during pregnancy and the postpartum period, and this formed the basis of the recommendation of the Centers for Disease Control and Prevention to defer isoniazid preventive therapy to the postpartum period in women without HIV infection. ${ }^{18,32-34}$ However, more recent small studies involving women who became pregnant while receiving isoniazid preventive therapy or who started receiving isoniazid preventive therapy during pregnancy did not show increased adverse events. ${ }^{28-30}$ We reviewed seven trials of isoniazid preventive therapy in nonpregnant adults in which liver function was monitored, albeit with variable algorithms and with variable proportions of participants who were receiving ART (Table S7 in the Supplementary Appendix). ${ }^{10-13,35-37}$ Whereas in our trial in pregnant women, $6.6 \%$ of participants had hepatotoxicity of grade 3 or higher (including four events of symptomatic hepatitis and two deaths [0.2\%] due to liver failure) while receiving isoniazid, studies in nonpregnant women showed lower percentages of participants with hepatotoxicity of grade 3 or higher, ranging from 1.1 to $2.9 \%$ in five trials totaling 8084 participants and from 4.0 to $5.5 \%$ in smaller trials involving less than 400 participants. Furthermore, in five trials, no deaths or a small number of deaths due to liver failure were reported in groups that received isoniazid ( 0 to $0.05 \%)^{10,12,13,35,36,38}$; in two trials, deaths were reported, but they were of unknown cause, and the relatedness to the trial drug was unclear. ${ }^{11,37}$

In the current trial, the majority of elevations in liver-enzyme levels of grade 3 or higher and all cases of symptomatic hepatitis and death occurred during the postpartum period; this highlights the importance of postpartum monitoring and follow-up. Some of these events were not considered to be associated with isoniazid, and all these events occurred in participants who were receiving efavirenz-based ART. No other cause of maternal death from liver failure (e.g., hepatitis A, hepatitis B, hepatitis C, or acetaminophen use) could be identified. It is possible that efavirenz - the first-line ART in $85 \%$ of our participants and a drug that has been associated with hepatotoxicity of grade 3 or higher or drug discontinuation in $2.3 \%$ of patients ${ }^{39}$ and fulminant hepatic failure in women ${ }^{40}-$ contributed to liver failure and death. An interaction between efavirenz and isoniazid may be a contributing factor, because isoniazid appears to inhibit an accessory pathway of efavirenz metabolism, which 
results in higher efavirenz levels, particularly in women who have slow metabolizing genotypes for both drugs (e.g., $\mathrm{N}$-acetyltransferase 2 slow acetylator and CYP2B6 TT). ${ }^{41}$

The higher incidence of the composite adverse pregnancy outcome among women in the immediate group than in the deferred group was a new finding. When examined separately, there were no significant between-group differences in any of the adverse pregnancy outcomes, although the frequencies of stillbirth or spontaneous abortion and low birth weight in an infant were numerically higher in the immediate group than in the deferred group. Studies in animals have shown that animals that receive at least twice the therapeutic dose of isoniazid have smaller litter sizes and offspring with a lower birth weight than those in comparison groups. ${ }^{42-44}$ Although the adverse pregnancy outcomes in this trial could be due to chance, given the large number of secondary outcomes that were analyzed, our results highlight a safety concern that warrants further examination.

This trial had limitations. We excluded women who were in the first trimester of pregnancy, and we did not evaluate the effect of isoniazid on organogenesis. We also excluded women with recent tuberculosis exposure - a group in which there is a higher risk of tuberculosis. Our noninferiority margin was just reached, and the results of the primary analysis were not robust to assumptions regarding missing data. Most participants were receiving efavirenz, and other antiretroviral agents may have a different riskbenefit profile. We observed low rates of tuberculosis, most likely because all participants received ART and isoniazid, because we used systematic baseline screening for tuberculosis, and because not all participants had latent tuberculosis infection (only $30 \%$ had latent infection at entry). Finally, the trial included monthly monitoring of liver-enzyme levels and other safety measures, rather than just monitoring of symptoms, which is the standard of care in current HIV and tuberculosis programs because of resource constraints as well as controversy regarding the clinical importance of asymptomatic elevated liver-enzyme levels. ${ }^{31}$ It is possible that we misclassified transient hepatic adaptation (a nonprogressive injury to hepatocyte cell structures) as liver injury because of our more intensive monitoring of laboratory values. Conversely, our frequent monitoring and management of elevated liver-enzyme levels may have averted severe hepatotoxicity.

The combination of isoniazid preventive therapy and ART is effective in reducing the risk of HIV-associated tuberculosis. ${ }^{11,14,36}$ In women with HIV infection who were receiving ART, the initiation of isoniazid preventive therapy during pregnancy was noninferior to initiation during the postpartum period with respect to maternal treatment-related adverse events. However, our finding of a greater incidence of adverse pregnancy outcomes in the immediate group than in the deferred group without any additional benefit with respect to the risk of tuberculosis or maternal or infant death is a cause for concern. Shortercourse regimens (such as isoniazid and rifapentine administered for 1 month or 3 months ${ }^{10,12,13,35,36,38}$ ) initiated during pregnancy may have different risk-benefit profiles, and further study is warranted. Our trial highlights the need to include pregnant women in clinical trials to inform global health policy. ${ }^{8,9}$

\footnotetext{
The content of this article is solely the responsibility of the authors and does not necessarily represent the official views of the National Institutes of Health.

A data sharing statement provided by the authors is available with the full text of this article at NEJM.org.

Supported by the National Institutes of Health (NIH) (award numbers, UM1AI068632 [IMPAACT LOC], UM1AI068616 [IMPAACT SDMC], and UM1AI106716 [IMPAACT LC]) through the National Institute of Allergy and Infectious Diseases, with cofunding from the Eunice Kennedy Shriver National Institute of Child Health and Human Development (contract number, HHSN275201800001I) and the National Institute of Mental Health.

Disclosure forms provided by the authors are available with the full text of this article at NEJM.org.

We thank the IMPAACT Network and operations staff for their support; the numerous community advisory boards, international site investigators, and research teams (Enid Kabugho, Hellen Kaganzi, Hajira Kataike, Joel Maena, and Deo Wabwire [Uganda]; Vongai Chanaiwa, Patricia Mandima, Suzen Maonera, Emmie Marote, Sukunena Maturure, Tapiwa Mbengeranwa, Tsungai Mhembere, Mercy Mutambanengwe, and Teacler Nematadzira [Zimbabwe]; Nasreen Abrahams, Haseena Cassim, Jeanne Louw, Ruth Mathiba, Sharon Mbaba, Mandisa Nyati, Karen du Preez, Lindie Rossouw, Magdel Rossouw, Marlize Smuts, Celeste de Vaal, and Frieda Verheye-Dua [South Africa]; Philoteus Sakasaka, Seleman Semvua, and Aisa Shao [Tanzania]; Unoda Chakalisa, Tebogo J. Kakhu, Ayotunde Omoz-Oarhe, and Thuto Ralegoreng [Botswana]; Neetal Nevrekar, Sandesh Patil, and Nishi Suryavanshi [India]; Prapaporn Janjing, Chintana Khamrong, Tavitiya Sudjaritruk, and Fuanglada Tongprasert [Thailand]; Maria Linda Aristhomene, Emelyne Dumont, Dominique Lespinasse, and Marie Flore Pierre [Haiti]; Soyeon Kim, Amy
} 
James Loftis, David Shapiro, and Camlin Tierney [IMPAACT]; more details are provided in the Supplementary Appendix); the women and families who participated in the trial; Vandana Kulkarni, who served as the protocol laboratory technologist; Joan Coetzee, who was a field representative; Rebecca LeBlanc, who coordinated sample transfer and data management at the central laboratory; Vivian Rexroad, who assisted in pharmacy training; Renee Browning, who was a protocol medical officer; the members of the independent end-point review committee; and Timothy R. Sterling, who represented the Tuberculosis Trials Consortium, which is funded by the Centers for Disease Control and Prevention.

\section{APPENDIX}

The authors' full names and academic degrees are as follows: Amita Gupta, M.D., M.H.S., Grace Montepiedra, Ph.D., Lisa Aaron, M.S., Gerhard Theron, M.D., Katie McCarthy, M.P.H., Sarah Bradford, M.P.H., Tsungai Chipato, M.B., Ch.B., F.R.C.O.G., M.C.E., Tichaona Vhembo, M.B., Ch.B., M.P.H., Lynda Stranix-Chibanda, M.B., Ch.B., M.M.E.D., Carolyne Onyango-Makumbi, M.B., Ch.B., M.S., Gaerolwe R. Masheto, M.D., Avy Violari, M.D., Blandina T. Mmbaga, M.D., M.M.E.D., Ph.D., Linda Aurpibul, M.D., M.P.H., Ramesh Bhosale, M.D., Vidya Mave, M.D., D.T.M.\&H., Vanessa Rouzier, M.D., Anneke Hesseling, M.D., Ph.D., Katherine Shin, Pharm.D., Bonnie Zimmer, B.S., Diane Costello, B.S., Timothy R. Sterling, M.D., Nahida Chakhtoura, M.D., Ms.G.H., Patrick Jean-Philippe, M.D., and Adriana Weinberg, M.D.

The authors' affiliations are as follows: the Center for Clinical Global Health Education, Johns Hopkins University, Baltimore (A.G., V.M.), and the Division of AIDS, National Institute of Allergy and Infectious Diseases (K.S., P.J.-P.), and the Eunice Kennedy Shriver National Institute of Child Health and Human Development (N.C.), National Institutes of Health, Bethesda - all in Maryland; the Harvard T.H. Chan School of Public Health, Boston (G.M., L. Aaron, G.R.M.); the Family Clinical Research Unit, Department of Obstetrics and Gynaecology, Stellenbosch University, Cape Town (G.T.), the Perinatal HIV Research Unit, University of the Witwatersrand, Johannesburg (A.V.), and the Desmond Tutu TB Center, Department of Paediatrics and Child Health, Stellenbosch University, Tygerberg (A.H.) - all in South Africa; FHI 360, Durham, NC (K.M., S.B.); University of Zimbabwe College of Health Sciences Clinical Trials Research Centre, Harare (T.C., T.V., L.S.-C.); Makerere University-Johns Hopkins University Research Collaboration, Kampala, Uganda (C.O.-M.); Botswana Harvard AIDS Institute Partnership, Gaborone (G.R.M.); Kilimanjaro Christian Medical Centre, Moshi, Tanzania (B.T.M.); Research Institute for Health Sciences, Chiang Mai University, Chiang Mai, Thailand (L. Aurpibul); Byramjee Jeejeebhoy Government Medical College (R.B.) and Byramjee Jeejeebhoy Government College-Johns Hopkins Clinical Research Site (A.G., V.M.), Pune, India; Les Centres GHESKIO Clinical Research Site (GHESKIO-INLR), Port au Prince, Haiti (V.R.); Frontier Science Foundation, Amherst, NY (B.Z.); University of California, Los Angeles, Los Angeles (D.C.); Vanderbilt University Medical Center, Nashville (T.R.S.); and the University of Colorado Denver Anschutz Medical Campus, Aurora (A.W.).

\section{REFERENCES}

1. Global tuberculosis report 2018. Geneva: World Health Organization, 2018 (http:// www.who.int/tb/publications/global _report/en/).

2. Jana N, Vasishta K, Saha SC, Ghosh K. Obstetrical outcomes among women with extrapulmonary tuberculosis. N Engl J Med 1999;341:645-9.

3. Mathad JS, Gupta A. Tuberculosis in pregnant and postpartum women: epidemiology, management, and research gaps. Clin Infect Dis 2012;55:1532-49.

4. Pillay T, Khan M, Moodley J, Adhikari M, Coovadia H. Perinatal tuberculosis and HIV-1: considerations for resource-limited settings. Lancet Infect Dis 2004;4:155-65. 5. Gomes VF, Andersen A, Wejse C, et al. Impact of tuberculosis exposure at home on mortality in children under 5 years of age in Guinea-Bissau. Thorax 2011;66 163-7.

6. Gupta A, Nayak U, Ram M, et al. Postpartum tuberculosis incidence and mortality among HIV-infected women and their infants in Pune, India, 2002-2005. Clin Infect Dis 2007;45:241-9.

7. Frigati L, Bekker A, Stroebele S, Goussard P, Schaaf HS. Culture-confirmed tuberculosis in South African infants younger than 3 months of age: clinical presentation and management of respiratory complications. Pediatr Infect Dis J 2019;38: 351-4.
8. Gupta A, Mathad JS, Abdel-Rahman $\mathrm{SM}$, et al. Toward earlier inclusion of pregnant and postpartum women in tuberculosis drug trials: consensus statements from an international expert panel. Clin Infect Dis 2016;62:761-9.

9. McKenna L, Frick M, Lee C, et al. A community perspective on the inclusion of pregnant women in tuberculosis drug trials. Clin Infect Dis 2017;65:1383-7.

10. Martinson NA, Barnes GL, Moulton LH, et al. New regimens to prevent tuberculosis in adults with HIV infection. N Engl J Med 2011;365:11-20.

11. Rangaka MX, Wilkinson RJ, Boulle A, et al. Isoniazid plus antiretroviral therapy to prevent tuberculosis: a randomised double-blind, placebo-controlled trial. Lancet 2014;384:682-90.

12. Samandari T, Agizew TB, Nyirenda $S$, et al. 6-Month versus 36-month isoniazid preventive treatment for tuberculosis in adults with HIV infection in Botswana: a randomised, double-blind, placebo-controlled trial. Lancet 2011;377:1588-98. 13. Sterling TR, Villarino ME, Borisov AS, et al. Three months of rifapentine and isoniazid for latent tuberculosis infection. N Engl J Med 2011;365:2155-66.

14. Latent TB infection: updated and consolidated guidelines for programmatic management. Geneva: World Health Organization, 2018 (https://www.who.int/ tb/publications/2018/latent-tuberculosis -infection/en/).

15. Frederiksen MC. Physiologic changes in pregnancy and their effect on drug disposition. Semin Perinatol 2001;25:120-3. 16. Anderson GD. Pregnancy-induced changes in pharmacokinetics: a mechanistic-based approach. Clin Pharmacokinet 2005;44:989-1008.

17. Kourtis AP, Read JS, Jamieson DJ. Pregnancy and infection. N Engl J Med 2014;371:1077.

18. Franks AL, Binkin NJ, Snider DE Jr, Rokaw WM, Becker S. Isoniazid hepatitis among pregnant and postpartum Hispanic patients. Public Health Rep 1989; 104:151-5.

19. Moulding T. Isoniazid-associated hepatitis deaths: a review of available information. Am Rev Respir Dis 1992;146: 1643-4.

20. Ouyang DW, Shapiro DE, Lu M, et al. Increased risk of hepatotoxicity in HIVinfected pregnant women receiving antiretroviral therapy independent of nevirapine exposure. AIDS 2009;23:2425-30.

21. QuantiFERON-TB Gold (in-tube method). Germantown, MD: Qiagen (package insert) (https://www.quantiferon.com/us/ products/quantiferon-tb-gold/technical -resources/).

22. Cherry CL, Wesselingh SL, Lal L, McArthur JC. Evaluation of a clinical 
screening tool for HIV-associated sensory neuropathies. Neurology 2005;65:1778-81. 23. National Institute of Allergy and Infectious Diseases, Division of AIDS (DAIDS). Table for grading the severity of adult and pediatric adverse events. Version 2. November 2014 (https://rsc.tech-res .com/docs/default-source/safety/daids_ae _grading_table_v2_nov2014.pdf?sfvrsn=8). 24. Metropolitan Atlanta Congenital Defects Program (MACDP). Atlanta: Centers for Disease Control and Prevention (https:// www.cdc.gov/ncbddd/birthdefects/macdp .html).

25. Greenland S, Robins JM. Estimation of a common effect parameter from sparse follow-up data. Biometrics 1985;41:55-68. 26. Jennison C, Turnbull BW. Group sequential methods with applications to clinical trials. Boca Raton, FL: Chapman \& Hall/CRC Press, 2000.

27. Lancaster H. Significance tests in discrete distributions. J Am Stat Assoc 1961; 56:223-34.

28. Moro RN, Scott NA, Vernon A, et al. Exposure to latent tuberculosis treatment during pregnancy: the PREVENT TB and the iAdhere trials. Ann Am Thorac Soc 2018;15:570-80.

29. Taylor AW, Mosimaneotsile B, Mathebula U, et al. Pregnancy outcomes in HIVinfected women receiving long-term isoniazid prophylaxis for tuberculosis and antiretroviral therapy. Infect Dis Obstet Gynecol 2013;2013:195637.
30. Tiam A, Machekano R, Gounder CR, et al. Preventing tuberculosis among HIVinfected pregnant women in Lesotho: the case for rolling out active case finding and isoniazid preventive therapy. J Acquir Immune Defic Syndr 2014;67(1):e5-e11.

31. Saukkonen JJ, Cohn DL, Jasmer RM, et al. An official ATS statement: hepatotoxicity of antituberculosis therapy. Am J Respir Crit Care Med 2006;174:935-52.

32. American Thoracic Society. Targeted tuberculin testing and treatment of latent tuberculosis infection. MMWR Recomm Rep 2000;49(RR-6):1-51.

33. Moulding TS, Redeker AG, Kanel GC. Twenty isoniazid-associated deaths in one state. Am Rev Respir Dis 1989;140:700-5. 34. Snider DE Jr, Caras GJ. Isoniazid-associated hepatitis deaths: a review of available information. Am Rev Respir Dis 1992; 145:494-7.

35. Swindells S, Ramchandani R, Gupta A, et al. One month of rifapentine plus isoniazid to prevent HIV-related tuberculosis. N Engl J Med 2019;380:1001-11.

36. Menzies D, Adjobimey M, Ruslami R, et al. Four months of rifampin or nine months of isoniazid for latent tuberculosis in adults. N Engl J Med 2018;379:44053.

37. The TEMPRANO ANRS 12136 Study Group. A trial of early antiretrovirals and isoniazid preventive therapy in Africa. N Engl J Med 2015;373:808-22.

38. Sterling TR, Scott NA, Miro JM, et al.
Three months of weekly rifapentine and isoniazid for treatment of Mycobacterium tuberculosis infection in HIV-coinfected persons. AIDS 2016;30:1607-15.

39. Shubber Z, Calmy A, Andrieux-Meyer I, et al. Adverse events associated with nevirapine and efavirenz-based first-line antiretroviral therapy: a systematic review and meta-analysis. AIDS 2013;27:1403-12. 40. Sonderup MW, Maughan D, Gogela N, et al. Identification of a novel and severe pattern of efavirenz drug-induced liver injury in South Africa. AIDS 2016;30: 1483-5.

41. Dooley KE, Denti P, Martinson N, et al. Pharmacokinetics of efavirenz and treatment of HIV-1 among pregnant women with and without tuberculosis coinfection. J Infect Dis 2015;211:197-205.

42. Bharathi KN, Natesh TS, Ashwitha Reddy A. Prenatal exposure to anti-tubercular drugs and postnatal effect on growth, development and cognitive ability in rats. Prog Neuropsychopharmacol Biol Psychiatry 2012;37:203-9.

43. Brost BC, Newman RB. The maternal and fetal effects of tuberculosis therapy. Obstet Gynecol Clin North Am 1997;24: 659-73.

44. Toxicology Data Network. Isoniazid: human health effects. Bethesda, MD: National Library of Medicine (https:// toxnet.nlm.nih.gov/cgi-bin/sis/search/ a?dbs+hsdb:@term+@DOCNO+1647).

Copyright $(2019$ Massachusetts Medical Society. 